\title{
Simultaneous Demonstration of Microscopic Anatomy of Brainstem, Nissl Substance, Lipofuscin and Cytological Changes Using a Combination Method
}

\author{
Isan K. Patro*, Nisha Patro*, Praveena Singhal and Suresh K. Bhardwaj \\ Neurobiology and Ageing Unit, School of Studies in Zoology, Jiwaji University, Gwalior 474011, India. \\ (*Present address: MRC Neurochemical Pathology Unit, Newcastle General Hospital, \\ Newcastle upon Tyne, NE4 6BE, UK)
}

Received for publication July 24, 1995 and in revised form October 3, 1996

The proposed combination of three histochemical methods facilitates the demonstration of microscopic anatomy, Nissl content of neurons, lipofuscin accumulation and associated cytopathological changes, if any, in one brain section. Formaldehyde fixed paraffin sections were stained with a modified Weil's method, cresyl violet and pyronin $Y$ in sequence, dehydrated, cleared and mounted in DPX. The slides were studied under a fluorescence microscope. The fibre system stained grey. The Nissl substance and lipofuscin stained violet and pink, respectively. On exposure to UV light, the pink stained pigment emitted golden-yellow fluorescence, resembling the auto-fluorescence of unstained lipofuscin. The final results were remarkably reproducible.

Key words: Lipofuscin, Nissl substance, Brain anatomy, Cresyl violet

\section{Introduction}

Involvement of lipofuscin in brain aging is of complex nature. A similar or perhaps the same pigment also accumulates in cells under experimental conditions and in various diseased states. Thus studies on the role of the pigment in neuro-physiology and pathology have gained importance $[1,4,6-10,12,13,18]$. It is difficult to simultaneously demonstrate microscopic anatomy of brain regions, Nissl content and lipofuscin in the same section or a set of neurons, precisely because all conventional methods need different sections. This has been a limiting factor in establishing the involvement of pigment accumulation in neuron loss or other age associated cellular changes in brain. We have standardised a combination of three methods to simultaneously demonstrate the microscopic anatomy of brain slices, distribution pattern of Nissl substance and lipofuscin content in the same section, with the help of a microscope equipped with bright field and epi-fluorescence.

Correspondence to: Dr. I. K. Patro, MRC Neurochemical Pathology Unit, Newcastle General Hospital, Newcastle upon Tyne, NE4 6BE, UK. (After February, 1997 to my Indian address, please)

\section{Materials and Methods}

Three senile Wistar rats (30-32 months-old) and three senile Balb/C mice (18 month-old) were anaesthetised with ether vapours and perfused with 10\% chilled buffered formalin. Subsequently, their brains were removed and postfixed in $10 \%$ buffered formalin ( $\mathrm{pH} \mathrm{7.2)} \mathrm{for} 48 \mathrm{hr}$, washed under running water (overnight), dehydrated through a series of graded alcohol, cleared in toluene and embedded in paraffin wax (temp. $60-62^{\circ} \mathrm{C} ; 3-4 \mathrm{hr}$ ). Serial sections were cut at $10 \mu \mathrm{m}$ and stretched on clean and high quality glass slides. Sections of brainstem through pons region were used for standardising this method as it provides an unique combination of fibre system and the various nuclei. The sections were deparaffinised, hydrated and stained with Weil's haematoxylin (freshly prepared just before use; $10 \%$ haematoxylin in $95 \%$ ethanol further diluted to $1 \%$ with distilled water plus equal volume of $4 \%$ ferric ammonium sulphate) for $30 \mathrm{~min}$ at $45^{\circ} \mathrm{C}$, washed in 3 changes of water, 2 min each; differentiated in $4 \%$ ferric ammonium sulphate solution till the cellular areas become light grey; quickly washed in 2 changes of water; further differentiated in a mixture of $20 \%$ borax and $25 \%$ potassium ferrocyanide (1:1; 2-3 dips) and then washed in water for $5 \mathrm{~min}$. The Weil's haematoxylin stained sections were further stained with cresyl violet (cresyl violet 
$0.1 \mathrm{~g}$, distilled water $100 \mathrm{ml}$, glacial acetic acid $0.31 \mathrm{ml}$, and sodium acetate $0.0205 \mathrm{~g}$, adjusted to $\mathrm{pH}$ 3.5) for 3$6 \mathrm{~min}$ and then washed in distilled water. Subsequently, the sections were stained with $1 \%$ aqueous pyronin Y (extracted with chloroform) for $1 \mathrm{~min}$; washed in water (2-3 dips); air dried; quickly dehydrated in 2 changes of ethanol and cleared in xylene (when left for longer duration gives better results). Finally the sections were mounted in DPX. Some sections were stained similarly but with crystal violet instead of cresyl violet for a comparison. A third set of sections were stained only with cresyl violet and pyronin $\mathrm{Y}$ as mentioned above.

A set of sections were treated with $0.08 \%$ ribonuclease at $37^{\circ} \mathrm{C}(1 \mathrm{hr})$ or $10 \%$ perchloric acid at $4^{\circ} \mathrm{C}$ (overnight, followed by washing in $1 \%$ sodium carbonate) and then stained with cresyl violet and pyronin Y separately, as well as in combination. Untreated sections were also stained with cresyl violet, crystal violet or pyronin Y, separately, for comparison.

The sections were viewed under a Leitz fluorescence microscope equipped with epi-fluorescence. The fluorescence attachment consisted of a high pressure HOB $100 \mathrm{~W}$ mercury lamp and excitation filters with transmission maximum between $370-460 \mathrm{~nm}$ or $420-490 \mathrm{~nm}$ and barrier filter of $420 \mathrm{~nm}$.

\section{Results}

The myelinated fibre system of the brainstem and Nissl staining of the neurons made it convenient to reach a particular nucleus based on their cellular pattern more specifically and easily. While the fibre tracts stained grey, the neurons were easily identified by their Nissl content (Figs. 1-3). The other requirement is to localise lipofuscin and Nissl substance in the same neuron(s). We have recorded that the staining of the pigment with cresyl violet is relatively more intense in older animals as compared to the young [16]. Thus it is, at times, difficult to distiguish between the pigment and Nissl substance. On exciting the stained sections under UV or near UV light, a fading of cresyl violet staining became apparent in the pigment. It took 1-5 min to fade out the dye from lipofuscin and a relatively slower fading occurred in the Nissl substance.

When the sections were stained in pyronin $\mathrm{Y}$ following Weil's haematoxylin and cresyl violet staining, a shift
Table 1. Stainability of lipofuscin with various histochemical methods

\begin{tabular}{|c|c|c|c|}
\hline Stain & Nissl & Pigment & Fibres \\
\hline Weil & - & - & $\begin{array}{c}\text { Grey } \\
H\end{array}$ \\
\hline Cresyl violet & $\begin{array}{c}\text { Violet } \\
\text { H }\end{array}$ & $\begin{array}{l}\text { Violet } \\
+(1-5 \mathrm{~min})\end{array}$ & - \\
\hline $\begin{array}{l}\text { Cresyl violet + } \\
\text { Pyronin Y }\end{array}$ & $\begin{array}{c}\text { Violet } \\
\mathrm{H}\end{array}$ & $\begin{array}{l}\text { Pink to red } \\
+(1-5 \mathrm{~min})\end{array}$ & - \\
\hline $\begin{array}{l}\text { Pyronin Y after } \\
\text { treatment with } \\
\text { ribonuclease or } \\
\text { perchloric acid }\end{array}$ & - & $\begin{array}{l}\text { Pink to red } \\
\text { \# (No fading) }\end{array}$ & - \\
\hline Crystal violet & $\begin{array}{c}\text { Purple } \\
\text { HI }\end{array}$ & $\begin{array}{l}\text { Purple } \\
\text { H (10-15 min) }\end{array}$ & - \\
\hline Pyronin Y & $\begin{array}{c}\text { Pink } \\
\quad+\end{array}$ & $\begin{array}{l}\text { Pink to red } \\
\text { H }\end{array}$ & - \\
\hline $\begin{array}{l}\text { Crystal violet + } \\
\text { Pyronin Y }\end{array}$ & $\begin{array}{c}\text { Purple } \\
\text { HI }\end{array}$ & $\begin{array}{l}\text { Purple } \\
\text { H (10-15 min) } \\
\text { pink after fading }\end{array}$ & - \\
\hline
\end{tabular}

- Negative; + Mildly positive; + Intensely positive. Time required for fading of the stain under UV-light, in parentheses.

of colour of the pigment from violet to pink was noted. The pigment bodies in ribonuclease or perchloric acid treated sections when stained with either cresyl violet or pyronin $\mathrm{Y}$ or both the dyes together stained appreciably.

On exposure to UV light a fading of cresyl violet occurred (both in the pigment and the Nissl substance) but the pigment retained the pyronin Y staining (Figs. 4-8). The fading of the cresyl violet staining of the pigment was significantly faster than that of the Nissl substance. The fluorescence emitted by the pigment was similar to what was observed before pyronin staining or in the unstained preparations of the same tissue. This step also helped in demonstrating cytological changes associated with aging like chromatolysis and increased appearance of 'dark' cells [15].

While lipofuscin and Nissl stained differentially with cresyl violet, crystal violet stained both to a similar intensity. In addition crystal violet over stained the Weil's haematoxylin stained sections and was difficult to differen-

Fig. 1. A section through the pons of mice, stained with Weil's haematoxylin and cresyl violet allowing localisation of various nuclei, e.g. the nuclei lateral vestibularis (arrow head) and raphe obscurus (arrow), and the fibre systems have stained grey. $B a r=400 \mu \mathrm{m}$.

Figs. 2, 3. Higher magnification of the nuclei lateral vestibularis and raphe obscurus, respectively. Bar $=30 \mu \mathrm{m}$.

Fig. 4. A neuron from nucleus facialis of senile rat stained with cresyl violet and pyronin $Y$. The Nissl substance is stained violet and the lipofuscin (arrow) pink. Bar $=25 \mu \mathrm{m}$; same for Figs. 4-8.

Fig. 5. The neuron at Fig. 4 under epi-fluorescence. The lipofuscin pigment emitted golden-yellow autofluorescence (arrow).

Fig. 6. A portion of the nucleus facialis of rat brain stained with the three staining procedures (Weil's haematoxylin, cresyl violet and pyronin Y) simultaneously. The fibres are stained grey, the Nissl substance violet (arrows) and lipofuscin pink (arrow heads).

Fig. 7. The same set of neurons (as in Fig. 6) under fluorescence microscope. The lipofuscin pigment presents golden-yellow autofluorescence (arrow heads).

Fig. 8. The same area seen in Fig. 7 after exposure to UV-light. Note the retention of cresyl violet staining of the Nissl. 

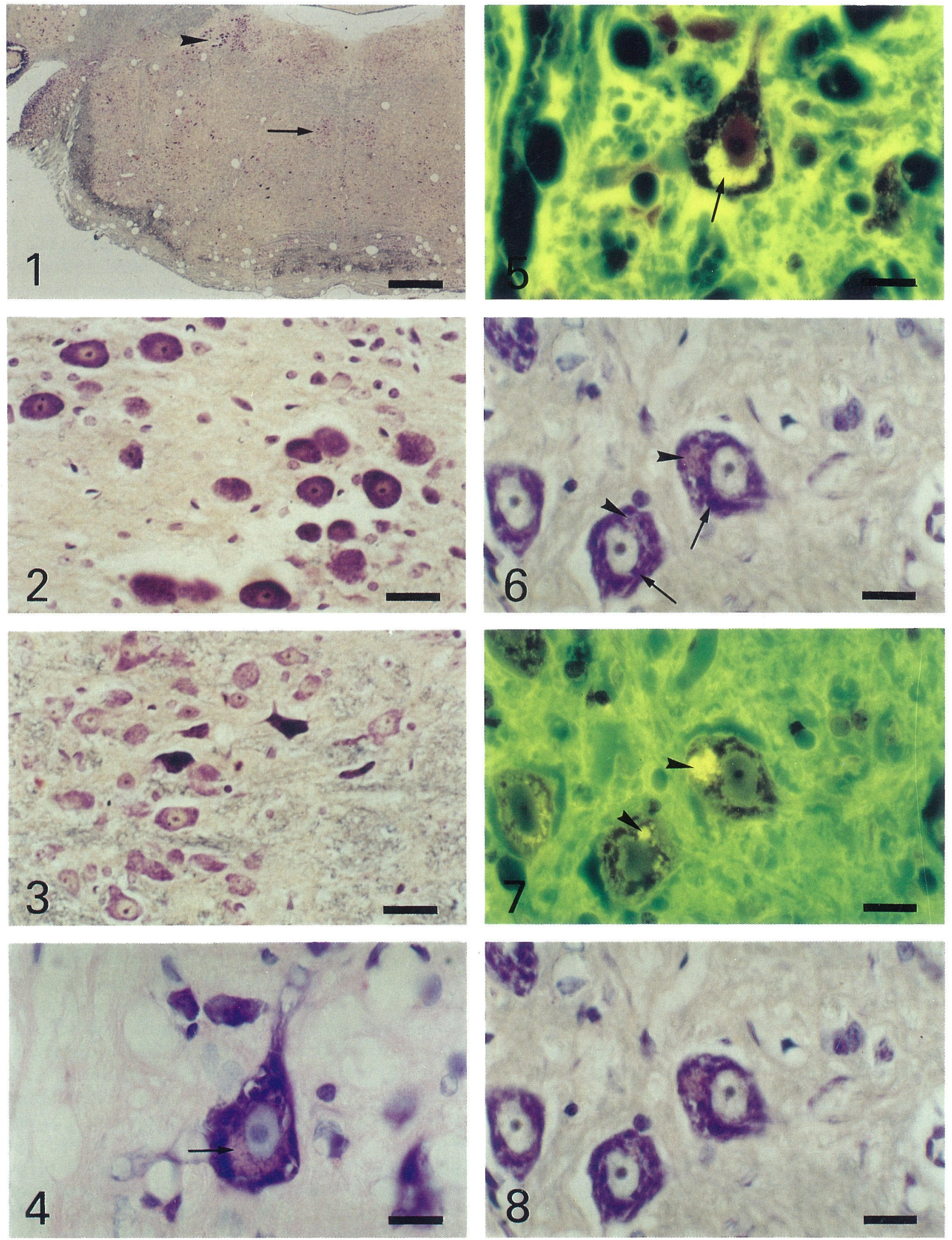

Figs. 1-8. 
tiate. Over and above more than $15 \mathrm{~min}$ exposure to UV light was necessary to fade out the staining from the lipofuscin (Table 1).

The tissues from both rats and mice presented strikingly similar results.

\section{Discussion}

Neurons get differentiated early in life and are one of the most suitable cells to record the age associated cellular changes. Intraneuronal accumulation of lipofuscin with age has been widely evidenced in a wide range of animals and man. An extensive accumulation of lipofuscin in neurons and other cell types has been considered detrimental to cell function. In addition recent studies have evidenced an inverse relationship between lipofuscin accumulation and cellular metabolism [8-10]. Some of the other prominent cytological changes in senile neurons are chromatolysis, pyroninophobe vacuolar spaces in both cytoplasm and nucleus, and loss of Nissl [13] as well as an increased population of 'dark' cells $[5,15]$.

Loss of neurons in several parts of aging brain has been widely accepted. Loss of Nissl and accumulation of lipofuscin are prominent in the areas of the brain presenting a significant decline in neuron population with age have been suggested. However, direct evidence on the relationship between rate of lipofuscin accumulation with neuron loss remains to be established. The conventional histological and histochemical methods need more than one section to demonstrate the microscopic anatomy of the brain regions, Nissl content of the neurons, lipofuscin accumulation and some of the associated cytological changes.

The proposed combination method was tried on brainstem sections as they present an unique combination of the fibre systems and the various nuclei. It shall however, work equally well on sections from other parts of the brain.

The method of Weil is simple and a reliable modification of Weigert-Pal methods. The principle of the Weigert-Pal method is: myelin reduces the chrome salts to chromium dioxide that acts as a mordant and forms a lake with haematoxylin. A modification was necessary because tissues treated with Weigert's primary mordant are of little value as they become brittle and cannot be stained with other histological procedures. In Weil's method the mordant and the dye are mixed in the staining solution and is thus preferred to the Weigert-Pal methods [2].

Most of the stains used for Nissl staining viz., methylene blue, toluidine blue, thionin, gallocyanin and cresyl violet also stain lipofuscin in paraffin sections. Lipofuscin has an affinity with thiazine (azure A, toluidine blue) and oxazine (cresyl violet, Nile blue sulphate) dyes giving an orthochromatic coloration [3, 11]. This suggests the presence of the carboxylic groups, sulphatides and possible presence of 1,3-diketones and enol groups. Intense basophilia is a sign of pigment maturation. The intensity deepens with the age of the animal [11]. It may even be weak to negative [14] or extremely variable in the spontaneous ceroids [3]. Elleder [3] holds that such confusion appears due to the use of a lowly standardised approach and the varied types of dyes being used which prevent proper comparison of the published results. Most of the dyes used for this purpose are lipophilic in nature. There is a possibility of a purefy physical dissolution of the dye molecules in the lipid phase of the pigment.

Cresyl violet has been recorded to differentially stain the Nissl bodies and lipofuscin, when further stained with pyronin $\mathrm{Y}$ a shift of the colour in lipofuscin provides a further advantage in distinguishing the pigment from Nissl. The colour visible at the site of pigment aggregates is perhaps due to the staining or retention of the dye in either the matrix or the space between the duplexes or the lipid phase of the pigment. Replacement of one dye by another has also been reported by Terr [17]. Nissl bodies being rich in RNA present affinity with many basic dyes including pyronin $\mathrm{Y}$ [14]. The changes in the colour of lipofuscin could be due to replacement of cresyl violet by pyronin Y. The replacement is faster at the site of pigment deposition than Nissl bodies which help in obtaining a preparation in which lipofuscin stains pink and Nissl bodies are still violet. The pigment in ribonuclease or perchloric acid treated sections stained intensely with pyronin Y suggesting thereby the involvement of substances other than RNA for the pyroninophility of the pigment. Thus staining could be simply due to a physical dissolution of the stain in the lipid phase of the lipofuscin pigment.

Terr [17] used crystal violet to demonstrate Nissl bodies and lipofuscin without assigning any reason for the choice of crystal violet. Our observations in the comparative study indicate that the use of cresyl violet would be more reproducible and informative than crystal violet. We propose this because: (a) Crystal violet tends to over stain sections stained with Weil's haematoxylin and it stains lipofuscin pigment to similar intensity as it does with Nissl bodies thus making it, at times, impossible to locate the pigment under bright field. (b) On exposure to UV or near UV light after crystal violet staining, the pigment characteristically fluoresces orange to golden yellow, but needs longer duration of exposure for fading of the dye. A shift in the colour of the pigment occurs, in the sections stained with pyronin $\mathrm{Y}$, from violet to pink resembling that of pyronin Y staining. (c) The differential staining of lipofuscin is more important and reproducible in a combination of cresyl violet and pyronin Y. (d) Differentiation of crystal violet staining is more crucial than cresyl violet in relation to fading of the dye and autofluorescence of lipofuscin. This is because the pigment stains more intensely with crystal violet than cresyl violet. In view of the above, the use of cresyl violet is advocated to demonstrate Nissl substance and lipofuscin simultaneously.

The final results of the present procedure are 
remarkably reproducible. Simultaneous demonstration of changes in microscopic anatomy, lipofuscin accumulation, Nissl content and even quantitation of neuron loss during aging and other experimental conditions are possible with this method in the same set of sections with an advantage over the conventional methods.

\section{Acknowledgments}

Financial assistance of the Department of Science and Technology, Govt. of India (Sanction No. SP/SO/ B44/90), is thankfully acknowledged. NP and SKB are recipients of grants from Department of Science and Technology, New Delhi and University Grants Commission, New Delhi, respectively.

\section{References}

1. Brizzee, K. R. and Ordy, J. M.: Cellular features, regional accumulation and prospects of modification of age pigment in mammals. In "Age Pigments", ed. by R. S. Sohal, Elsevier, North-Holland Biomedical Press, Amsterdam, 1981, pp. 101154.

2. Cox, G.: Neuropathological techniques. In "Theory and Practice of Histological Techniques", ed. by J. D. Bancroft and A. Stevens, Churchill Livingstone, Edinburgh, 1977, pp. 332-363.

3. Elleder, M.: Chemical characterisation of age pigments. In "Age Pigments", ed. by R. S. Sohal, Elsevier, North-Holland Biomedical Press, Amsterdam, 1981, pp. 203-241.

4. Hasan, M. and Glees, P.: Genesis and possible dissolution of neuronal lipofuscin. Gerontologie (Basel) 18; 217-236, 1972.

5. James, T. J., Sharma, S. P., Gupta, S. K. and Patro, I. K.: 'Dark' cell formation under protein malnutrition: Process of conversion and concept of 'semi-dark' type Purkinje cells. Indian J. Expt. Biol. 30; 470-473, 1992.

6. Jolly, R. D., Dalefield, R. R. and Palmer, D. N.: Ceroid, lipofuscin and ceroid lipofuscinosis (Batten disease). $J$. Inher. Metab. Dis. 16; 280-283, 1993.

7. Mann, D. M. A., Yates, P. O. and Stamp, J. E.: The relationship between lipofuscin pigment and ageing in the human nervous system. J. Neurol. Sci. 37; 83-93, 1978.

8. Patro, I. K. and Sharma, S. P.: Cytochemical interaction of nucleus and cytoplasm in the Purkinje cells of senile white rats under the influence of centrophenoxine. Exp. Gerontol. 19(4); 241-252, 1984.

9. Patro, I. K. and Patro, N.: Lipofuscin in ageing brain-A selective reappraisal. Indian Rev. Life Sci. 12; 133-145, 1992.

10. Patro, I. K., Sharma, S. P. and Patro, N.: Neuronal lipofuscin: Its formation and reversibility. Indian Rev. Life Sci. 8; 95-120, 1988.

11. Patro, I. K., Sharma, S.P. and Patro, N.: Formation and maturation of neuronal lipofuscin. Proc. Natl. Acad. Sci. (India) 59(B); 287-293, 1989.

12. Patro, I. K., Patro, N., Sharma, S. P. and Chaudhary, A.: Lipofuscinolysis: An overview. In "Advances in Physiological Sciences”, ed. by S. K. Manchanda, W. Selvamurthy and V. Mohan Kumar, Macmillan India, New Delhi, 1991, pp. 441450.

13. Patro, N., Patro, I. K. and Mathur, R.: Changes in properties of cardiac lipofuscin with age and environmental manipulation. Asian J. Exp. Sci. 7; 57-60, 1993.

14. Pearse, A. G. E.: Histochemistry: Theoretical and Applied, Vol. 2, 3rd ed., J. \& A. Chuchill, Ltd., London, 1972.

15. Sharma, S. P., Patro, I. K., Patro, N. and James, T. J.: 'Dark' type Purkinje cells and neuronal ageing. Proc. Indian Acad. Sci. (Anim. Sci.) 97(5); 449-453, 1988.

16. Singhal, P.: Formation and removal of lipofuscin and its role in neuron loss in brainstem of ageing mice. Ph. D. Thesis, Jiwaji University, Gwalior, India, 1996.

17. Terr, L. I.: Demonstration of lipofuscin and Nissl bodies in crystal violet stained sections using a fluorescence technique on pyronin Y stain. Stain Technol. 61(5); 309-313, 1986.

18. Wolf, G.: Lipofuscin, the age pigment. Nutr. Rev. 51(7); 205-206, 1993. 